\title{
Prospective Directions for the Development of Surface Plasmon Resonance Studies in Ukraine
}

\section{Maslov VP'*, Dorozinska HV'2 Ushenin $\mathrm{YV}^{1}$ and Dorozinsky $\mathbf{G V}^{\mathbf{2}}$}

${ }^{1} \mathrm{~V}$ Lashkaryov Institute of Semiconductor Physics NAS of Ukraine, Kyiv, Ukraine

${ }^{2}$ National Technical University of Ukraine "Igor Sikorsky Kyiv Polytechnic Institute", Kyiv, Ukraine

\begin{abstract}
Main centers for the development of applied plasmonics in Ukraine are Institute of Cybernetics and Lashkaryov Institute of Semiconductor Physics. The most significant advantage of biosensors "Plasmon" series produced by Lashkaryov Institute of Semiconductor Physics are block-modular design, which makes it easy to update the device for various applications and possibility investigation gaseous media. Original technical solutions allowed making quite inexpensive and affordable analytical device for researches in Ukraine, yet comparable in detection limit $\left(3 \times 10^{-6} \mathrm{RIU}\right)$ and accuracy $\left( \pm 1 \times 10^{-6} \mathrm{RIU}\right)$ with the most applicable instruments from the world's leading manufacturers. Wide measuring range from 1,0 to 1,5 RIU allowed study liquids with high refractive index like motor oils and polymers. So, promising areas of development of SPR-devices are increasing their accuracy, improving the technology of manufacturing a sensitive element and developing algorithms for processing results. This makes it possible to expand the use of SPR in medicine, microbiology and industry.
\end{abstract}

Keywords: Surface plasmon resonance; Sensors; Medicine; Industry

\section{Introduction}

One of the promising optical methods for analysis of various compounds and micro-objects as well as processes at the molecular level is the refractometric method based on surface plasmon resonance (SPR) phenomenon. Over the last two decade, SPR refractometry became one of the major methods for the detection and investigation of affinity based interactions in biochemistry, bioanalytical chemistry and biomedicine. Based on the SPR phenomenon refractometric method has been successfully used for the analysis s of the optical properties of a wide range of substances, from gases (e.g., anesthetic gases) [1] to liquids (e.g., analysis of the binary system of methanol in water), [2] and the solids (e.g., inorganic solid particles [3] and the organic film Langmuir-Blodgett [4]). In principle, SPR sensors are thin-film refractometers that measure changes in the refractive index occurring at the surface of a metal film supporting a surface plasmon [5]. However, from the sensing point of view, signal recording based on the change of thickness of the adjacent layer, resulting from formation of an affinity complex, is much more interesting. A surface plasmon excited by a light wave propagates along the metal film, and its evanescent field probes the medium (sample) in contact with the metal film. A change in the refractive index or thickness of the dielectric gives rise to a change in the propagation constant of the surface plasmon, which through the coupling condition alters the characteristics of the light wave coupled, e.g., angle. Since having been made commercially available by Swedish company Biacore AB (presently GE Healthcare) in 1990 [6], the SPR-based techniques have revolutionized the whole field of molecular biology. The most significant advantage of these techniques is their capability of label-free real-time monitoring of biomolecular interactions that opened up the way to develop a new concept for analysis of functional properties of biomolecules, the affinity biosensor technology. The SPR based technique can be applied to the measurement of the ligand-receptor and enzyme-substrate interactions, for label-free immunoassays, in polyclonal antibody characterization, epitope mapping, protein conformation studies, DNA hybridization etc. Apart from biomedical researches, it can also be used in the field of material science for studying interfacial physical and chemical processes: electrochemical reactions, corrosion, adsorption, heterogeneous catalysis and many others. The SPR biosensor advantage as compared with more conventional methods, for instance the enzyme linked immunoassay (ELISA), consists in the real-time monitoring of intermolecular interactions and performing fast analysis without need of enduring sample incubation.

In Ukraine, there are two main centers of the National Academy of Sciences of Ukraine (NASU) for the development of applied plasmonics: the Institute of Cybernetics and Lashkaryov Institute of Semiconductor Physics (ISP NASU). By Institute of Cybernetics under the direction of Dr.Sc., Prof. I. D. Voitovich basic model of the portable device based on a divergent beam of polarized radiation, an optical prism, a lens system and a photodiode CCD line was developed. The peculiarity of SPR diagnostics is the speed of analysis and high sensitivity. The absence of moving elements to change the angle of incidence of the beam and the use of sensor substrates with high adhesion provide high reliability of the device [7].

Experimental researches of applied aspects of the construction of biosensors based on SPR phenomenon at the Lashkaryov Institute of Semiconductor Physics of the National Academy of Sciences of Ukraine (ISP NASU) were initiated in the early 90s under the direction of Dr.Sc., Prof. Yu. M. Shirshov. The core of the SPR research group was formed in the late 90s as a part of the research team working on an international INCO-Copernicus project devoted to the development of biosensors. Nowadays, the research team from ISP NASU based on the Department of physical and technological bases of sensory materials lead by Dr.Sc., Prof. Volodymyr P. Maslov and the SPR group led by Senior Researcher Yuri V. Ushenin.

*Corresponding author: Maslov VP, V Lashkaryov Institute of Semiconductor Physics NAS of Ukraine, Kyiv, Ukraine, Tel: +38(044) 525-58-30; E-mail: vpmaslov@ukr.net

Received August 21, 2017; Accepted September 22, 2017; Published October 25, 2017

Citation: Maslov VP, Dorozinska HV, Ushenin YV, Dorozinsky GV (2017) Prospective Directions for the Development of Surface Plasmon Resonance Studies in Ukraine. J Laser Opt Photonics 4: 166. doi: 10.4172/2469-410X.1000166

Copyright: (c) 2017 Maslov VP, et al. This is an open-access article distributed under the terms of the Creative Commons Attribution License, which permits unrestricted use, distribution, and reproduction in any medium, provided the original author and source are credited. 
Constructively completed model of the SPR refractometer (Plasmon-3), which is suitable for use in biochemical and biophysical laboratories, was developed at the end of the nineties (year 1998). Highly qualified physicists, programmers and instrument builders were involved in this project, which in the future formed the core of the research and development group, which continued the further development of SPR refractometry and its applications in scientific research. Biosensor "Plasmon-5" was first serially capable one-channel biosensor based on surface plasmon resonance, which was developed at the ISP NASU. The later was created double-channel refractometr "Plasmon-6" (year 2004). The device was created during the work of the model series "Plasmon", which were successfully used in many laboratories in Ukraine and abroad. Today, in ISP NASU was developed the dual-channel refractometers "Plasmon-6", "Plasmon-7", "Plasmon-71", "Plasmon-9" and eight-channel device "Plasmon-8". One of the designed models "Plasmon-71" allows you to measure the refractive index over a wide range from 1 to 1.5 RIU (refractive index unit) with detection limit $\pm 3 \times 10-6$ RIU and accuracy $\pm 1 \times 10-6$ RIU. All devices features supplementary electronic channel for recording data from external equipment (like potentiostat in electrochemical studies) synchronously with the SPR data. Accessories also include thermo stabilized flow-cell (up to $95^{\circ} \mathrm{C}$ ), syringe and peristaltic pump. The "Plasmon" series are versatile measuring devices designed for application in various industries and field of research, running with PCs and having complex and versatile software. The developed software allows maximum use of the capabilities of devices when working in laboratory conditions. Versions of the device can be capable of longterm autonomous operation, which provides the ability to work in the village fields. Devices may have an autonomous power supply and specialized built-in software aimed at solving specific tasks. The most significant advantage of "Plasmon" devices is small dimensions, low weight (less than $3 \mathrm{~kg}$ ), block - modular design, which makes it easy to update the device for various applications and possibility investigation gaseous media.

In this review we present prospective directions and applying for the development of surface plasmon resonance studies in Ukraine.

\section{Application SPR Technique in Medicine and Biology}

The SPR group collaborates on a permanent basis with practice in the areas of biology and medicine. One of the most promising results was obtained during investigations of blood cells agglomeration conducted jointly with the Romodanov Institute of Neurosurgery of the National Academy of Medical Science of Ukraine. A correlation has been revealed between SPR response to peripheral blood samples of different patients and severity of diseases from ageing to craniocerebral trauma and grade III-IV gliomas [8]. These observations were explained by differences in modes of agglomeration of erythrocytes absorbed upon the sensor surface. The development of an innovative SPR-based experimental technique with "Plasmon-9" contributed also to the clinical practice [9]. Additional important achievements were related to the field of emergency medicine and express care. It involved development of an SPR-based technique for fast monitoring of thrombus formation. Critical parameters for thrombus formation are concentration of fibrinogen or fibrin degradation product in patient's blood [10]. A portable immunosensor analyser of thrombus-related species based on SPR-refractometer model "Plasmon-8", intended for use in medical practice as part of equipment for medical emergency teams.

The major practical applications of SPR biosensors are related to medical diagnostics and they are directly related to improvement of health care in Ukraine. Among other investigations related to medical applications of SPR- based techniques and worth mentioning here is the research for development of the immunosensor for detection of the Epstein-Barr virus (EBV), carrying out at the Zabolotny Institute of Microbiology and Virology of NASU (IMV) [11]. EBV is a virus of the herpes family also called human herpesvirus 4 (HHV-4). This virus has been found to be implicated in several diseases from relatively mild infectious mononucleosis to several forms of cancer, such as Burkitt's lymphoma, Hodgkin's lymphoma and nasopharyngeal carcinoma. There is evidence that it is also associated with a higher risk of certain autoimmune diseases like multiple sclerosis. Current investigations are aimed at increasing both sensitivity and reliability of EBV antibodies detection. Evaluation of treatment efficiency for inhibition of proliferation and inducing apoptosis of cancer cells by nanoforms of plant polyphenols using "Plasmon-6" has been performed at the Kavetsky Institute of Experimental Pathology, Oncology and Radiology of NASU [12]. Rats with passaged Walker rat carcinosarcoma and Ca755 mouse carcinoma were used in this study. Content of transcription factor NF-kB and peptides produced by dependent genes in extracts derived from tumors in laboratory animals amenable to this treatment was measured using SPR method and immunoblotting. It has been shown that the results obtained by SPR method were consistent with those obtained from more conventional techniques and correlated well with biological effects observed in vivo.

The SPR-based sensors possess exquisite sensitivity for the determination of DNA hybridization [13]. Determination of the serum level of immunoglobulins is very important for the diagnosis of autoimmune and immunodeficiency diseases [14]. By Ukrainian scientists has been performed the successful immobilization of recombinant protein A from Staphylococcus aureus with the C-terminal cysteine residue (SPA-Cys) on the "Plasmon-6" nanolayer sensor surface while preserving its high immunoglobulin-binding activity and selectivity of the sensor response. The suitability of SPA-Cys as a bioselective component for the creation of the biosensor for the IgG detection has been shown [15].

Another area of research refers to the veterinary medicine, namely to the development of SPR-based method of bovine leucosis diagnostics. This disease is caused by the bovine leukemia virus (BLV) discovered in 1969. Since BLV was found to contain an oncogene coding, a search for BLV pathogenicity humans started soon after its discovery. However, no conclusive evidence of BLV transmission to human was found till now. Later studies have revealed high prevalence of BLV infection among dairy herds in many countries, including Ukraine. It is generally believed that high susceptibility to this type virus is genetically predisposed and resulted from genetic transformation in some breeds of high producing dairy cows, especially Holstein cattle. Since BLV dissemination has become a global threat to cattle breeding, considerable efforts were put into eradication of this disease throughout the world [16]. Among a variety of proposed measures, availability of reliable methods for BLV diagnosis is of great importance. At present, BLV diagnosis relies mainly upon the serological methods for detection of the presence of specific antibodies in BLV infected cattle, the Agar Gel immune diffusion (AGID) and Enzyme-Linked Immunosorbent Assay (ELISA), and/or on the Polymerase Chain Reaction (PCR) capable of revealing the viral RNA (or proviral DNA) in serum. In Ukraine investigates the ways to BLV diagnosis using optical methods, in particular, with the Plasmon SPR biosensors [17]. They have demonstrated that sensitivity of SPR method is sufficient for detection of BLV-specific antibodies in milk instead of serum, thus simplifying the sampling procedure. 
Thus, the potential of advanced SPR to improve cost-efficiency and speed of medical diagnostics and treatment is obvious.

\section{Application SPR Technique in Industry}

Diagnosing the devices working on the phenomenon of SPR is highly sensitive to low concentrations of analytes, allowing them to be used not only as a gas analyzer [18-20], but also as high-precision analytical instruments for laboratory analysis in chemical and pharmaceutical industry and environment control [21]. Lubricants are widely used in up-to-date technology for lowering friction in movable mechanisms of engines, bearings, gears and so on. One of the first-rate consumer using motor oils is automotive industry. When taking into account a wide field of lubricant applications, there occurs a problem to examine their quality in the course of production and operation. Enhancing the ecological standards causes increasing the fraction of oils with energy- saving and biodegradation properties, which lowers the operation life of these motor oils. Thus, there arises the task of simultaneous determining both the condition of motor oil and degree of car engine wear, with high sensitivity and productivity. This task can be solved using highly precise fast examining lubricants, which can be provided by the measurement of optical properties inherent to motor oils with application of SPR-technique. In work [22] the SPR method was applied to determine refraction indexes of pure and used (three thousand kilometres mileage) synthetic motor oil Mobil Super 3000 fe sw-30. It was shown that there are essential differences between these two oil samples both in refraction indexes and in character of their changes in time. The refraction index of pure oil was increased with time, while that of the used one was increased, which was explained by the effect of temperature factor and sedimentation of the oxidation products onto a sensitive element of the measuring device. Authors in study [23] carried out the measurements of the refraction indexes for four samples of synthetic motor oil genuine $5 \mathrm{w}-30$ dexos 2, namely: before using it in the car engine (fresh) as well as after its operation for the mileages 180, 430 and $712 \mathrm{~km}$ (used). They performed measurements of kinetics (changes in time) of the refraction index for the studied samples by using the SPR refractometer "Plasmon-71". In addition, they measured the same samples with the optical refractometer RL3. It has been experimentally shown that using the SPR method improves more than one order (from 0.17 vol.\% down to 0.0107 vol.\%) the detection limit and enhances sensitivity of measuring the wear particles concentration in motor oil as compared with the refractometric method. Thus, it is experimentally proved that the SPR method can be offered to control quality of motor oils as well as the degree of wear inherent to interacting parts of machinery.

Photopolymer curing adhesives are widely used for connecting optical instrument parts in the producing of precision optical devices. This connection method has several advantages compared with other methods of connection of optical components. As is well known, introduction into the adhesive mass low dispersion adhesive filler reduces shrinkage after polymerization reduces the internal stresses in the connection of optical components and increases the reliability of the connection. Moreover, the dispersion medium affects the polymerization rate increase which is technologically and economically advantageous. By varying the number and quality of the filler can vary the refractive index of the compound to achieve the best optical alignment of the parts. Thus, the problem arises of controlling the refractive index of photopolymer adhesives and their rate of polymerization depending on the quality and amount of filler. This task can be solved using highly precise fast examining UV-glues, which can be provided by the measurement of optical properties (polymerization speed and refractive index) with application of SPR-technique. Devices based on the SPR allow quickly determining the optical properties of photopolymer curing adhesives and monitoring processes in real time [24]. Researchers conducted by the photopolymer adhesives with various fillers have shown the effectiveness and feasibility of utilizing the phenomenon of SPR to monitor the optical parameters of photopolymer polymerization adhesives.

\section{Enhancing Accuracy Methods}

The most effective directions for improving the accuracy of SPR devices are as follows: increasing the wavelength of radiation exciting surface plasmons, modernization of technology for preparation of metal layer in the sensitive element and stabilization of temperature of the device and analyte during measurements. The investigations performed by the authors have shown that increasing the excitation wavelength also results in decreasing the error of determination analyte refraction index by 5.5 times: from $\pm 6.2 \bullet 10-5$ down to $\pm 1.1 \bullet 10-5$ [21] As it follows from experiments, roughness of the metal layer surface in sensitive element can be and reduced by 25 times (from 2 down to 0.8 $\mathrm{nm}$ ) due to updating technology of its thermal deposition in vacuum on a glass substrate, which increase sensitivity in 2 time and reduces the measurement error by 5.8 times (from $\pm 7 \cdot 10$-6 down to $\pm 1.2 \cdot 10-6$ ) [25]. In both cases, this decrease in errors is reached due to narrowing the minimum of the reflection characteristics, which, in turn, was caused by increasing the excitation wavelength and decreasing the roughness of the sensor elements metal layer. The decreases in temperature error during measurements of the analyte refraction index have been reached using stabilization of the temperature inherent to the device and reservoirs with analyte. In the case of gas-like analytes, the error has been 18-fold decreased (from 7•10-6 down to 3.8・10-7), while for the liquid ones - by 3.2 times (from $2.5 \bullet 10-5$ down to $7.6 \bullet 10$ 6) $[21,26,27]$.

The considered here additional methods for increasing the measurement accuracy are numeric methods of data processing based on calculations of the exact position of the reflection characteristic minimum for a finite value of the scanning step, when the true minimum value is located between adjacent steps. Our comparison of the most widely used methods has shown that the most exact method is that offered by the author (mean line method). It provides decreasing the measurement error by more than 60 times (from $2.4 \cdot 10$ - 4 down to $4 \cdot 10-6)$ as compared with measurements without any numeric processing the experimental data [28].

\section{Features Trends in SPR Technique}

For surface immobilization of biorecognition elements on the sensing Au layer, self-assembled monolayers (SAMs) of alkanethiolates or disulfides have been widely used [29]. The formed S-Au linkage is stable at air and water, but it decomposes under UV irradiation and at temperatures above $70^{\circ} \mathrm{C}$. Moreover, although dense monolayers assemble quickly, well-ordered monolayers can take days to form [30]. Therefore, the existing approaches are time-consuming and produce layers with low stability at elevated temperature and under UV irradiation. One of the most suitable candidates to substitute SAMs for biosensors surface modification is the plasma polymerization of ultrathin functional films (thickness of 5-20 nm). Such thin layers should not degrade the SPR formation on the one hand and enable efficient grafting of $\mathrm{COOH}, \mathrm{NH}_{2}$, anhydride or other reactive groups. Nowadays, plasma polymers were already employed for the biomolecular immobilization and cell adhesion improvement. Compared to SAMs, these coatings will be stable in a wider range of 
the conditions, are independent of the substrate material and will be deposited significantly faster and without use of solvents. Then, on the plasma-coated Au surface the antibody can be immobilized and the SPR biosensor will be able to detect the analyte. In work, Kovař et al. [31] were tested the (plasma polymer)-treated Au SPR chips for stability in different buffers sensory element with self-assembled monolayer were used for comparison. The tested sensor is slightly unstable in time. It seems that the layer is partially degraded or the non-reacted monomer/ oligomer molecules became gradually washed out. Considering very similar behavior in the reference flow channel, one can conclude that the polymer is stable in all tested buffers and the gradual loss of signal level is not dependent on $\mathrm{pH}$ within the tested interval.

\section{Conclusion}

Main centers for the development of applied plasmonics in Ukraine are Institute of Cybernetics and Lashkaryov Institute of Semiconductor Physics. The most significant advantage of biosensors produced by Lashkaryov Institute of Semiconductor Physics are small dimensions, low weight (less than $3 \mathrm{~kg}$ ), block-modular design, which makes it easy to update the device for various applications and possibility investigation gaseous media. Wide measuring range from 1,0 to 1,5 RIU allowed study liquids with high refractive index like motor oils and polymers. Original technical solutions implemented in SPRrefractometers "Plasmon" series allowed making quite inexpensive and affordable analytical device for researches in Ukraine, yet comparable in detection limit $(3 \times 10-6$ RIU $)$ and accuracy $( \pm 1 \times 10-6$ RIU $)$ with the most applicable instruments from the world's leading manufacturers. Innovative approaches to the sensor technology that was developed, namely structuring of the gold sensor surface, special numeric data processing methods, application in medicine and in industry, will further enhance the performance of Plasmon biosensors and will keep the Ukrainian research group at the forefront of sensor technologies.

So, promising areas of development of SPR-devices are increasing their accuracy, improving the technology of manufacturing a sensitive element and developing algorithms for processing results. This makes it possible to expand the use of SPR in medicine, microbiology and industry.

\section{References}

1. Liedberg B, Nylander C, Lundstrom I (1983) Surface Plasmon Resonance for Gas Detection and Biosensing. Sensors and Actuators 4: 299-304.

2. Matsubara K, Kawata S, Minami S (1988) Optical Chemical Sensor Based on Surface Plasmon Measurement. Appl Optics 27: 1160-1163.

3. Xu H, Kall M (2003) Modeling the optical response of nanoparticle-based surface plasmon resonance sensors. Sensors and Actuators 87: 244-249.

4. Rella R, Siciliano P, Quaranta F, Primo T, Valli L, et al. (2000) Gas Sensing Measurements and Analysis of the Optical Properties of Poly [3-(butylthio) thiophene] Langmuir-Blodgett films. Sensors and Actuators B 68: 203-209.

5. Homola J (2006) Surface Plasmon Resonance Based Sensors, Springer Series on Chemical Sensors and Biosensors, O. S.Wolfbeis, Springer- Verlag, Berlin Heidelberg.

6. Malmqvist M (1999) BIACORE: An affinity biosensor system for characterization of biomolecular interactions. Biochem Soc Trans 27: 335-340.

7. Voitovich I (2011) Sensors based on plasmon resonance: principles, technologies, Kiev, "Stal'- 53, p. (In Russian).

8. Gridina N, Maslov V, Ushenin Y (2013) Tumor-associated inflammation and brain glioma tumors. Lambert Academic Publishing, p: 196.

9. Gridina N, Dorozinsky G, Khristosenko R, Maslov V, Samoylov A, et al. (2013) Surface plasmon resonance biosensor. Sensors \& Transducers J 149: 60-68.

10. Snopok B, Kostyukevych K, Rengevych O, Shirshov Y, Venger E, et al. (2012) A biosensor approach to probe the structure and function of the adsorbed proteins: fibrinogen at the gold surface. Optoelec Semicond Tech 46: 45-49.
11. Khristosenko R, Nesterova N, Golovan A, Kostyukevich E, Zagorodnyaya $S$, et al. (2011) Immunosensor based on surface Plasmon resonance for determination of antibodies to the Epstein-Barr virus. Optoelectron Semicond Tech 46: 92- 99.

12. Samoylenko E, Zaletok S, Gogol S, Orlovskii A, Ushenin Yu, et al. (2012) Proceedings of V-th Int. Conf. "Sensor Electronics and Microsystem Technologies" (in Russian).

13. Rachkov A, Holodova Yu, Ushenin Yu, Miroshnichenko D, Telegeev G et al. (2009) Development of Bioselective Element of SPR Spectrometer for Monitoring of Oligonucleotide Interactions and Comparison with Thermodynamic Calculations. Sensor Letters 7: 45-52.

14. Notarangelo L, Fischer A, Geha R, Casanova J, Chapel H, et al. (2009) Primary immunodeficiencies: 2009 update. The International Union of Immunological Societies (IUIS) Primary Immunodeficiencies (PID) Expert Committee. J Allergy Clin Immunol 124: 1161-1178.

15. Bakhmachuk A, Gorbatiuk O, Rachkov A, Dons'koi B, Khristosenko R, et al (2017) Surface Plasmon Resonance Investigations of Bioselective Elemen Based on the Recombinant Protein A for Immunoglobulin Detection. Nanoscale Research Letters 12: 112.

16. Esteban E, Poli M, Poiesz B, Dube S, Gutierrez S, et al. (2009) Bovine leukemia virus (BLV), proposed control and eradication programs by marker assisted breeding of genetically resistant cattle. In: Animal Genetics, Leopold J editor. Rechi, Nova Science Publishers.

17. Pirogova L, Starodub N (2008) Immobilization of the antigen of the retroviruses of cattle on the surface of the immune biosensor. Biotechnology 2: 52-58.

18. Samoylov A, Mirsky V, Hao Q, Swart C, Shirshov Y, et al. (2005) Nanometerthick SPR sensor for gaseous HCl. Sensors and Actuators B Chem 106: 369-372.

19. Kostyukevych K, Khristosenko R, Shirshov Yu, Kostyukevych S, Samoylov A et al. (2011) Multi- element gas sensor based on surface plasmon resonance: recognition of alcohols by using calixarene films. Semiconductor Physics. Quantum Electronics and Optoelectronics 14(3): 313-320.

20. Dorozinsky G, Lobanov M, Maslov V (2015) Detection of methanol vapor by surface plasmon resonance method. Eastern-European Journal of Enterprise Technologies 4: 4-7.

21. Maslov V, Ushenin Yu, Dorozinsky G (2017) Trends in Improving the Accuracy of SPR-Devices. Sensors and Applications in Measuring and Automation Control Systems. Advances in Sensors: Reviews 4: 101-119.

22. Dorozinsky G, Liptuga A, Gordienko V, Maslov V, Pidgornyi V (2015) Diagnostics of motor oil quality by using the device based on surface plasmon resonance phenomenon. Scholars Journal of Engineering and Technology 3 372-374.

23. Maslov V, Dorozinsky G, Khrystosenko R, Samoylov A, Dorozinska, et al (2017) Surface plasmon resonance- a promising method for estimating the quality of motor oil. Trans \& Motauto World Journal 1: 41-44.

24. Dorozinsky G (2013) Surface plasmon resonance as a tool for research in nanotechnology and industry. Machin Tech Mat J 9: 51-54.

25. Dorozinsky G, Doroshenko T, Maslov V (2015) Influence of technological factors on sensitivity of analytical devices based on surface plasmon resonance. $J$ Sensor Tech 5: 54-61.

26. Dorozinsky G, Maslov V, Samoylov A, Ushenin Yu (2013) Reducing measurement uncertainty of instruments based on the phenomenon of surface plasmon resonance. Ame J Optics and Photo1: 17-22.

27. Ushenin Yu, Maslov V, Dorozinsky G, Turu T, Kachur N (2016) Application of temperature sensors for improving the device based on the phenomenon of surface Plasmon. Sensor Electronics and Microsystem Technologies 13: 33-40.

28. Maslov V, Ushenin Yu, Dorozinsky G, Dorozinska H, Kudryavtsev A (2017) New method for determining the angular position of the light reflection intensity minimum observed in surface plasmon resonance. J Multidisciplinary Eng Sci Stud 3: 1514-1518.

29. Lofas S (2007) Biosens. Bioelecton 22: 10-20.

30. Serenatne W (2005) Biomacromolecules 6: 24-27.

31. Kovař D, Makhneva E, Dorozinsky G, Manakhov A, Zajičková L, et al (2015) Study of carboxy-rich plasma polymer for biosensors. Proceedings of VII International conference on innovations in thin film processing and characterization. 